\title{
Ramucirumab as Second-Line Systemic Therapy in Hepatocellular Carcinoma
}

\author{
Masatoshi Kudo \\ Department of Gastroenterology and Hepatology, Kindai University Faculty of \\ Medicine, Osaka-Sayama, Japan
}

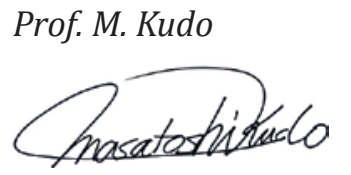

Editor Liver Cancer

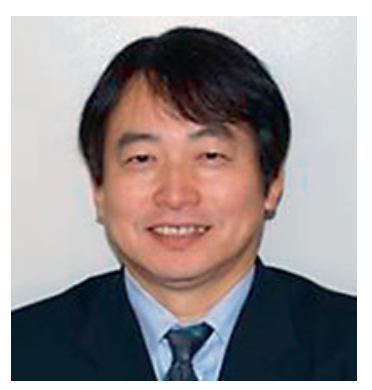

\section{Introduction}

Ramucirumab, a molecular targeted agent, is a recombinant human IgG1 monoclonal antibody directed against vascular endothelial growth factor receptor 2 (VEGFR-2) that plays an important role in vascular endothelial growth factor (VEGF)-induced tumor angiogenesis. It inhibits VEGFR-2 activation by blocking its binding to VEGF-A, VEGF-C, and VEGF-D, thereby inhibiting endothelial growth, migration, and survival and exerting antitumor effects via inhibition of tumor angiogenesis. Ramucirumab is currently indicated for unresectable advanced/ recurrent gastric cancer, colorectal cancer, and non-small-cell lung cancer and is used in routine clinical practice. A randomized phase III trial (REACH-2) investigated ramucirumab as secondline treatment following first-line sorafenib therapy for advanced hepatocellular carcinoma (HCC) in patients with an alpha-fetoprotein (AFP) level $\geq 400 \mathrm{ng} / \mathrm{mL}$, and results demonstrating the superiority of ramucirumab over placebo in overall survival (OS) were reported at the American Society of Clinical Oncology (ASCO) annual meeting in June 2018. This article outlines the results of the REACH-2 trial and previous clinical trials of ramucirumab (REACH).

\section{The REACH Trial}

Trial Design of REACH

Before discussing the REACH-2 trial, a preceding trial (the REACH trial) needs to be described. The REACH trial, like the REACH-2 trial, is a randomized phase III trial investigating ramucirumab as second-line treatment following first-line therapy with sorafenib in 
patients with advanced HCC; 146 centers across 26 countries participated in the trial [1]. The inclusion criteria for the REACH trial included history of sorafenib therapy, Barcelona Clinic Liver Cancer (BCLC) stage B/C, Child-Pugh class A, and Eastern Cooperative Oncology Group performance status (ECOG PS) score 0 or 1 . Patients were stratified by geographic region (North America, South America, Europe, and Eastern Asia) as well as underlying hepatic disease (infection with hepatitis B virus, hepatitis $C$ virus, and others) and randomly allocated to either the ramucirumab group or the placebo group in a 1:1 ratio. The primary endpoint was $\mathrm{OS}$, and the secondary endpoints were progression-free survival(PFS), time to progression (TTP), objective response rate (ORR), and safety. In the REACH trial, 565 patients were randomly allocated to the ramucirumab group (283 patients) or the placebo group (282 patients) between November 4, 2010 and April 18, 2013; patient characteristics were not markedly different between the two groups. Patients received ramucirumab $(8 \mathrm{mg} / \mathrm{kg})$ intravenously on day 1 of a 14-day cycle (ClinicalTrials.gov NCT01140347).

\section{Results of the REACH Trial}

The primary endpoint, OS, in the ramucirumab group was 9.2 months (95\% CI 8.0-10.6) while that in the placebo group was 7.6 months (95\% CI 6.0-9.3); the difference was not significant (HR $=0.87,95 \%$ CI $0.72-1.05, p=0.14$ ). In contrast, when a subgroup of patients with a baseline AFP level $\geq 400 \mathrm{ng} / \mathrm{mL}(n=250)$ was analyzed, OS in the ramucirumab group (119 patients) was 7.8 months (95\% CI 5.8-9.3) while that in the placebo group (131 patients) was 4.2 months (95\% CI 3.7-4.8), showing significant improvement of OS by ramucirumab (HR $=0.67,95 \%$ CI $0.51-0.90, p=0.006)$. Meanwhile, in a subgroup of patients with a baseline AFP level $<400 \mathrm{ng} / \mathrm{mL}(n=310)$, analysis showed that OS in the ramucirumab group (160 patients) was 10.1 months (95\% CI 8.7-12.3) while that in the placebo group (150 patients) was 11.8 months (95\% CI 9.9-13.1), showing no significant difference between the groups (HR $=1.09,95 \%$ CI 0.84-1.43, $p=0.51)$.

Although the difference in OS was not statistically significant, the difference in PFS was significant between the two groups (HR $=0.63,95 \%$ CI $0.52-0.75, p<0.0001$ ): 2.8 months (95\% CI 2.7-3.9) in the ramucirumab group vs. 2.1 months (95\% CI 1.6-2.7) in the placebo group. A significant difference in TTP was also found (HR $=0.59,95 \%$ CI $0.49-0.72, p<$ 0.0001): 3.5 months (95\% CI 2.8-4.5) in the ramucirumab group vs. 2.6 months $(95 \% \mathrm{CI}$ $1.6-2.8)$ in the placebo group. ORR was $7 \%$ (95\% CI 4.6-10.7) in the ramucirumab group, with objective response achieved by 20 patients ( 1 with complete response [CR] and 19 with partial response $[\mathrm{PR}])$ and $<1 \%(95 \% \mathrm{CI} 0.2-2.5)$ in the placebo group, with 2 patients achieving objective response (none with $\mathrm{CR}$ and 2 with PR), with significant intergroup difference $(p<0.0001)$. Grade $\geq 3$ severe adverse events occurring in $\geq 5 \%$ of patients were ascites ( $5 \%$ in the ramucirumab group vs. $4 \%$ in the placebo group), hypertension (12 vs. $4 \%$ ), asthma (5 vs. $2 \%$ ), progression of malignant neoplasm ( 6 vs. $4 \%$ ), elevated aspartate aminotransferase level ( 5 vs. $8 \%$ ), thrombocytopenia ( 5 vs. 1\%), hyperbilirubinemia (1 vs. $5 \%)$, and elevated bilirubin level (2 vs. $5 \%$ ).

\section{Analysis of the REACH Subgroups}

The results of the Japanese subgroup analysis of the REACH trial were reported by Kudo et al. [2]. Out of the 565 subjects in the REACH trial, 93 were Japanese, and among this population, OS differed significantly between the ramucirumab group (45 patients, 12.9 months) and the placebo group (48 patients, 8.0 months) (HR $=0.621,95 \%$ CI $0.391-0.986$, $p=0.0416)$. PFS was significantly longer in the ramucirumab group (4.1 months) than in the placebo group (1.7 months) (HR $=0.449,95 \%$ CI $0.285-0.706, p=0.0004)$. ORR was $11 \%$ in the ramucirumab group (no patient with CR, 5 with PR), but $2 \%$ in the placebo group (no patient with $\mathrm{CR}, 1$ with PR); the difference was not significant $(p=0.0817$ ) due to the 


\section{Liver
Cancer}

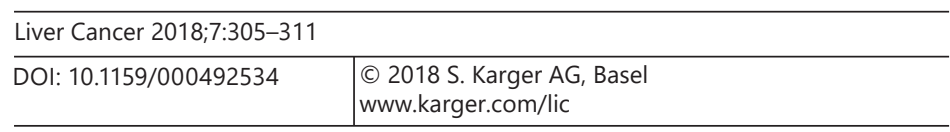

Kudo: Ramucirumab as Second-Line Systemic Therapy in Hepatocellular Carcinoma

small number of cases. Disease control rate (DCR) differed significantly ( $p=0.0462$ ) between the ramucirumab group (67\%; no patient with CR, 5 with PR, and 25 with stable disease [SD]) and the placebo group (46\%; no patient with CR, 1 with PR, and 21 with SD). There was no marked difference in therapy received after the REACH trial between the two groups. More adverse events were observed in the ramucirumab group than in the placebo group; grade $\geq 3$ severe adverse events occurring in $\geq 5 \%$ of patients were ascites $(7 \%$ in the ramucirumab group vs. $2 \%$ in the placebo group), hypertension (7 vs. $2 \%$ ), and cholangitis (7 vs. $0 \%$ ), suggesting sufficient tolerability of ramucirumab therapy in the Japanese population.

In Japanese patients with a baseline AFP level $\geq 400 \mathrm{ng} / \mathrm{mL}$ ( 20 and 22 patients in the ramucirumab group and the placebo group, respectively), OS was significantly different between the ramucirumab group (12.9 months) and the placebo group (4.3 months) (HR = $0.464,95 \%$ CI $0.232-0.926, p=0.0263$ ). Ramucirumab therapy prolonged OS by 8.6 months in patients with a baseline AFP level $\geq 400 \mathrm{ng} / \mathrm{mL}$. Meanwhile, in patients with a baseline AFP level $<400 \mathrm{ng} / \mathrm{mL}$ (25 and 26 patients in the ramucirumab group and the placebo group, respectively), the difference in OS between the former (12.9 months) and the latter group (12.4 months) was not significant (HR $=0.738,95 \%$ CI 0.391-1.394, $p=0.3492$ ).

As mentioned earlier, significant differences in OS between the ramucirumab group and the placebo group were found in the subgroup with an AFP level $\geq 400 \mathrm{ng} / \mathrm{mL}$ in the study population of the REACH trial and in the Japanese subgroup with an AFP level $\geq 400 \mathrm{ng} / \mathrm{mL}$. In addition, Park et al. [3] conducted a subgroup analysis of East Asian and non-East Asian populations. OS was prolonged by 3.4 months in the ramucirumab group (7.8 months) compared with the placebo group (4.2 months) in the East Asian population in patients with an AFP level $\geq 400 \mathrm{ng} / \mathrm{mL}$, although the difference was not significant (HR $=0.749,95 \% \mathrm{CI}$ $0.519-1.082, p=0.1213$ ). Conversely, in patients with an AFP level $\geq 400 \mathrm{ng} / \mathrm{mL}$, OS was significantly improved in the ramucirumab group ( 8.2 months) compared with the placebo group ( 4.5 months) in the non-East Asian population (HR $=0.579,95 \%$ CI $0.371-0.904, p=$ 0.0149). In patients with an AFP level $<400 \mathrm{ng} / \mathrm{mL}, \mathrm{OS}$ was not significantly different between the two groups, and no elongation of OS by ramucirumab therapy was observed in either population. Chau et al. [4] reported that the percent AFP increase was significantly lower and that decreases in AFP level and the percent tumor reduction were larger in the ramucirumab group than the placebo group: AFP progression and time to radiographic progression, which correlated with each other, were shorter in the ramucirumab group than in the placebo group.

\section{Summary of the REACH Trial}

Unfortunately, the REACH trial did not meet the primary endpoint in prolonging OS, although it showed no major safety issues. PFS, TTP, and ORR were significantly better in the ramucirumab group than in the placebo group. In patients with a baseline AFP level $\geq 400 \mathrm{ng} /$ $\mathrm{mL}$, ramucirumab significantly improved $\mathrm{OS}$, and several subgroup analyses confirmed the good outcome in this subgroup of patients. Thus, the REACH- 2 trial, focusing on patients with a baseline AFP level $\geq 400 \mathrm{ng} / \mathrm{mL}$, was conducted.

\section{The REACH-2 Trial}

\section{Trial Design of REACH-2}

The REACH-2 trial, like the REACH trial, is a randomized phase III trial investigating ramucirumab as second-line treatment following first-line sorafenib therapy in patients with advanced HCC; 131 centers across 20 countries participated in the trial. Based on the 


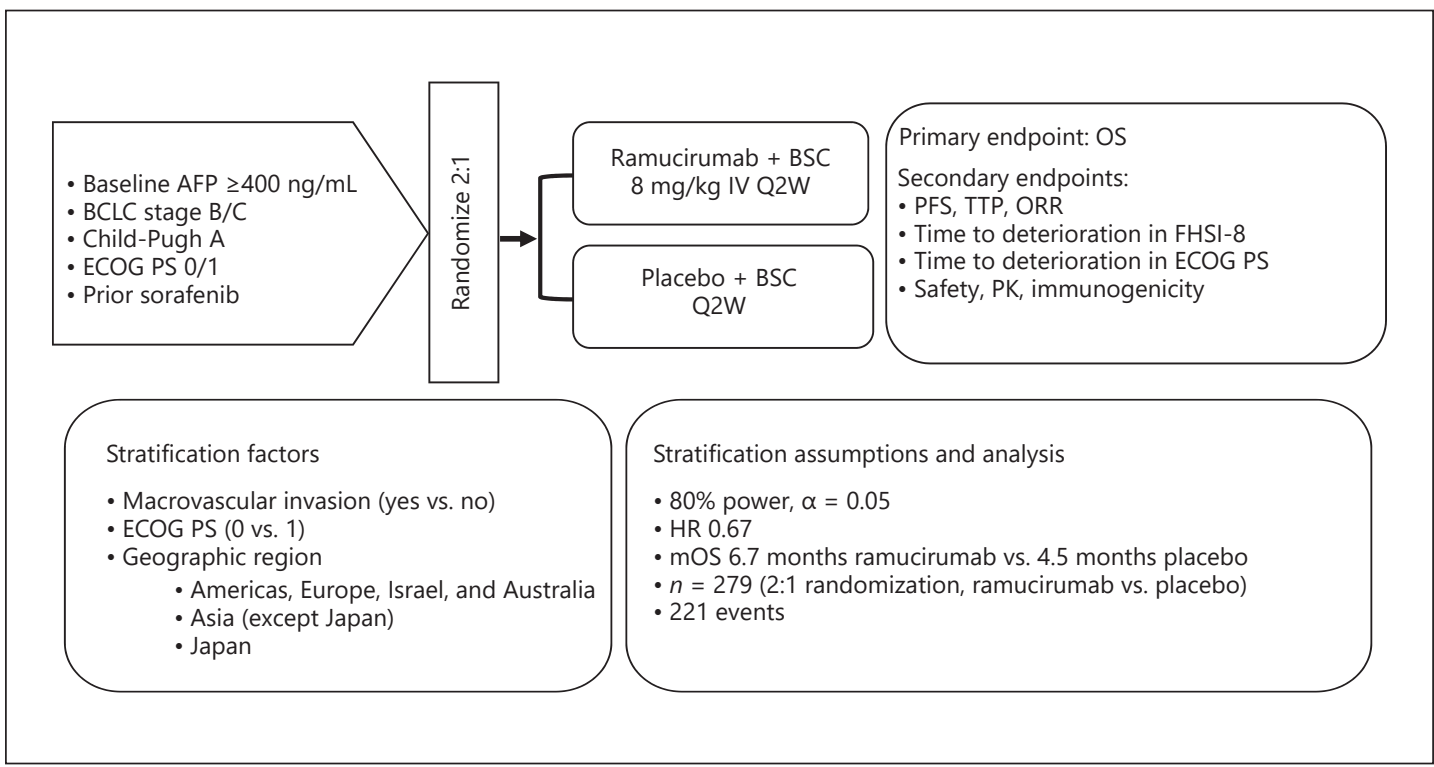

Fig. 1. Study design. ClinicalTrials.gov NCT02435433. Cited and modified from Zhu et al. [5]. AFP, alpha-fetoprotein; BCLC, Barcelona Clinic Liver Cancer; BSC, best supportive care; ECOG PS, Eastern Cooperative Oncology Group performance status; FHSI-8, Functional Assessment of Cancer Therapy Hepatobiliary Symptom Index-8; IV, intravenous; mOS, median overall survival; ORR, objective response rate; OS, overall survival; PFS, progression-free survival; PK, pharmacokinetics; Q2W, every 2 weeks; TTP, time to progression.

findings of the REACH trial, the new criterion of a baseline AFP level $\geq 400 \mathrm{ng} / \mathrm{mL}$ was added to the initial inclusion criteria of the REACH trial, including history of sorafenib therapy, BCLC stage B/C, Child-Pugh class A, and ECOG PS score 0 or 1 . A new stratification factor, macrovascular invasion, was also added, and patients were also stratified by geographic region (region 1 comprising North America, South America, Europe, Israel, and Australia, region 2 comprising Asian countries except Japan, and region 3 comprising Japan). Patients were stratified by ECOG PS score ( 0 or 1 ), but not by underlying liver disease (infection with hepatitis $B$ virus, hepatitis $C$ virus, and others), and were randomly allocated to either the ramucirumab group or the placebo group in a 2:1 ratio. The required number of 279 cases was calculated using the OS of 4.5 months and 6.7 months in the placebo group and the ramucirumab group (HR 0.67), respectively, detection power of $80 \%$, and $\alpha=0.05$ (two-sided). The primary endpoint was OS, and the secondary endpoints were PFS, TTP, ORR, safety, and pharmacokinetics. A total of 292 patients were randomly allocated to the ramucirumab group (197 patients) or the placebo group (95 patients). There were imbalances in favor of the placebo group: the median baseline AFP level was $3,920 \mathrm{ng} / \mathrm{mL}$ in the ramucirumab group and $2,741 \mathrm{ng} / \mathrm{mL}$ in the placebo group, and more patients had advanced HCC in the former than in the latter group. As with the REACH trial, patients received ramucirumab $(8 \mathrm{mg} / \mathrm{kg}$ ) intravenously on day 1 of a 14-day cycle (ClinicalTrials.gov NCT02435433) (Fig. 1).

\section{Results of the REACH-2 Trial}

The results of the REACH-2 trial were reported at the ASCO annual meeting in June 2018 [5]. OS was 8.5 months in the ramucirumab group and 7.3 months in the placebo group; the difference was significant ( $\mathrm{HR}=0.710,95 \%$ CI $0.531-0.949, p=0.0199$ ) (Table 1 ). Ramucirumab therapy decreased the mortality rate by $29 \%$. In all subgroups except for females, OS 
Table 1. Results of the REACH-2 trial

\begin{tabular}{|c|c|c|c|c|}
\hline & $\begin{array}{l}\text { Ramucirumab } \\
(n=197)\end{array}$ & $\begin{array}{l}\text { Placebo } \\
(n=95)\end{array}$ & HR & $p$ value \\
\hline Median overall survival & 8.5 months & 7.3 months & 0.710 & 0.0199 \\
\hline Median progression-free survival & 2.8 months & 1.6 months & 0.452 & 0.0001 \\
\hline Objective response rate & $4.6 \%$ & $1.1 \%$ & - & 0.1967 \\
\hline Relative dose intensity & $97.9 \%$ & $99.8 \%$ & - & - \\
\hline Discontinuation due to TEAE & $10.7 \%$ & $3.2 \%$ & - & - \\
\hline Dose adjustment due to $\mathrm{AE}$ & $34.5 \%$ & $13.7 \%$ & - & - \\
\hline
\end{tabular}

Cited and modified from Zhu et al. [5]. AE, adverse event; TEAE, treatment-emergent adverse event.

was longer in patients who received ramucirumab than in those who received placebo, particularly in men, those with extrahepatic metastases, and those without vascular invasion.

PFS was 2.8 months in the ramucirumab group and 1.6 months in the placebo group; the difference was significant (HR $=0.452,95 \%$ CI $0.339-0.603, p<0.0001$ ). Ramucirumabtreated patients had favorable PFS in all subgroups. ORR was $4.6 \%(95 \% \mathrm{CI} 1.7-7.5)$ with no CR case and 9 PR cases in the ramucirumab group, and $1.1 \%$ (95\% CI 0.0-3.1) with no CR case and 1 PR case in the placebo group; the difference was not significant $(p<0.1697)$ due to the small number of cases. DCR was significantly better in the ramucirumab group than in the placebo group ( $p=0.0006$ ): 59.9\% (118 cases comprising 0 CR, 9 PR, and 109 SD cases) in the former group and 38.9\% (37 cases comprising no CR, 1 PR, and 36 SD cases) in the latter group.

The median dose exposure in the ramucirumab group was 6 (range 3-13) cycles, while that in the placebo group was 4 (range 3-6) cycles. The relative dose intensity in the former was $97.7 \%$ while that in the latter was $99.8 \%$, indicating that ramucirumab therapy was uninterrupted in almost all cases. Ramucirumab targets a single molecule (VEGFR-2) and is likely to have fewer adverse events and favorable tolerability. The rates of study drug discontinuation due to adverse events were 10.7 and $3.2 \%$ in the ramucirumab group and the placebo group, respectively, and those of dose modification were 34.5 and $13.7 \%$, respectively. Grade $\geq 3$ adverse events occurring in $\geq 5 \%$ of patients were hypertension $(12.7 \%$ in the ramucirumab group vs. $5.3 \%$ in the placebo group), bleeding (5.1 vs. $3.2 \%$ ), and liver damage (18.3 vs. $15.8 \%)$.

\section{Interpretation of REACH-2 Trial Results}

REACH-2, which reexamined ramucirumab in patients with a baseline AFP level $\geq 400$ $\mathrm{ng} / \mathrm{mL}$ based on the results of the preceding REACH, was a positive study confirming significantly longer OS in ramucirumab- than in placebo-treated patients. PFS and DCR were also significantly better, indicating the drug's potency. The results regarding adverse events were similar to those shown in ramucirumab monotherapy for other indications, suggesting good tolerability in these patients. REACH-2 is an excellent trial because it is the first prospective randomized controlled biomarker-driven trial with positive outcomes.

Kaplan-Meier survival curves showed a significant difference in survival rate between the ramucirumab group (24.5\%) and the placebo group (11.3\%) at 18 months ( $p=0.0187)$, but not at 12 months. This can be explained by the imbalance between the two groups regarding the baseline AFP level (Table 2) and the proportion of BCLC stage C. Since both were higher in the ramucirumab group than in the placebo group, the effect of ramucirumab was not apparent until the later period. Furthermore, patients with a baseline AFP 


\section{Liver
Cancer}

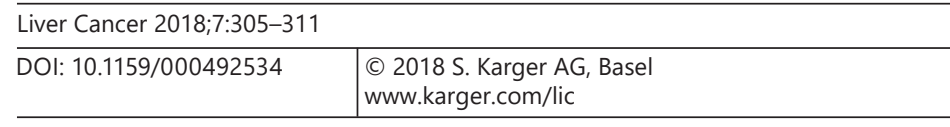

Kudo: Ramucirumab as Second-Line Systemic Therapy in Hepatocellular Carcinoma

Table 2. Comparison between REACH (AFP $\geq 400 \mathrm{ng} / \mathrm{mL}$ ), REACH-2, and pooled REACH-2/REACH (AFP $\geq 400 \mathrm{ng} / \mathrm{mL})$

\begin{tabular}{|c|c|c|c|c|c|c|}
\hline & \multicolumn{2}{|l|}{$\begin{array}{l}\text { REACH } \\
(n=250)\end{array}$} & \multicolumn{2}{|l|}{$\begin{array}{l}\text { REACH-2 } \\
(n=292)\end{array}$} & \multicolumn{2}{|c|}{$\begin{array}{l}\text { Pooled REACH-2/REACH } \\
(n=542)\end{array}$} \\
\hline & ramucirumab & placebo & ramucirumab & placebo & ramucirumab & placebo \\
\hline mOS, months & 7.8 & 4.2 & 8.5 & 7.3 & 8.1 & 5.0 \\
\hline HR (95\% CI) & \multirow{2}{*}{\multicolumn{2}{|c|}{$\begin{array}{c}0.674(0.508-0.895) \\
0.0059\end{array}$}} & \multirow{2}{*}{\multicolumn{2}{|c|}{$\begin{array}{c}0.710(0.531-0.949) \\
0.0199\end{array}$}} & \multirow{2}{*}{\multicolumn{2}{|c|}{$0.694(0.571-0.842)$}} \\
\hline$p$ value & & & & & & \\
\hline $\mathrm{mAFP}, \mathrm{ng} / \mathrm{mL}$ & $\mathrm{N} / \mathrm{A}$ & N/A & 3,920 & 2,741 & $4,104.6$ & $4,047.5$ \\
\hline
\end{tabular}

AFP, alpha-fetoprotein; mAFP, median alpha-fetoprotein; mOS, median overall survival; N/A, not available.

level $\geq 400 \mathrm{ng} / \mathrm{mL}$, which is associated with poor prognosis, might have died in the placebo group without having received post-progression treatment. Selection of trial subjects using the level of a biomarker (in this instance, AFP) contributed to the positive outcomes despite an enormously small number of patients $(n=292)$ for a clinical trial of a secondline agent.

Comparison of the results of the placebo group with a baseline AFP level $\geq 400 \mathrm{ng} / \mathrm{mL}$ showed that OS was 7.3 months in the REACH-2 trial, which was longer than the 4.2 months in patients with an AFP level $\geq 400 \mathrm{ng} / \mathrm{mL}$ in the REACH trial. This can be explained by the imbalance in patient characteristics regarding baseline AFP level mentioned earlier. The AFP value in the placebo arm (not presented anywhere) in the REACH trial (AFP $\geq 400 \mathrm{ng} / \mathrm{mL}$ ) must have been much higher than that in the placebo arms of REACH-2 $(2,741 \mathrm{ng} / \mathrm{mL})$ when considering the pooled data of REACH ( $\geq 400 \mathrm{ng} / \mathrm{mL})$ plus REACH-2 data $(4,047.5 \mathrm{ng} / \mathrm{mL})$ since the AFP value in the placebo arm in REACH-2 was too low as compared with that in the pooled data (Table 2). For the same reason, the HR for OS was slightly lower in the REACH-2 trial (0.67) than in patients with an AFP level $\geq 400 \mathrm{ng} / \mathrm{mL}$ in the REACH trial (0.71).

\section{Analysis of the REACH-REACH-2 Pooled Population}

The results of the analysis of 542 patients comprising 292 subjects of the REACH-2 trial and 250 subjects with an AFP level $\geq 400 \mathrm{ng} / \mathrm{mL}$ in the REACH trial were also reported at ASCO 2018. More precisely, OS was 3.1 months longer in the ramucirumab group ( 8.1 months) than in the placebo group (5.0 months); this difference was statistically significant (HR = $0.694,95 \%$ CI 0.571-0.842, $p=0.0002$ ) (Table 2). The AFP was well balanced in both arms in these pooled data $(4,104.6 \mathrm{ng} / \mathrm{mL}$ in the ramucirumab arm and $4,047.5 \mathrm{ng} / \mathrm{mL}$ in the placebo arm).

\section{Future Prospects of Ramucirumab in HCC Treatment}

Based on the results of REACH-2, ramucirumab will be indicated for advanced HCC as second-line treatment after sorafenib failure when the baseline AFP level is $\geq 400 \mathrm{ng} / \mathrm{mL}$. Approval of ramucirumab is awaited as it is likely to be beneficial in HCC patients with elevated AFP level who progressed on sorafenib or even on lenvatinib, and especially in patients intolerant to sorafenib who are not candidates for regorafenib. 
Kudo: Ramucirumab as Second-Line Systemic Therapy in Hepatocellular Carcinoma

\section{Disclosure Statement}

Masatoshi Kudo received lecture fees from Bayer, Eisai, MSD, and Ajinomoto, research grants from Chugai, Otsuka, Takeda, Taiho, Sumitomo Dainippon, Daiichi Sankyo, MSD, Eisai, Bayer, AbbVie, Medico's Hirata, Astellas Pharma, and Bristol-Myers Squibb, and advisory consulting fees from Kowa, MSD, BristolMyers Squibb, Bayer, Chugai, Taiho, Eisai, and Ono Pharmaceutical.

\section{References}

1 Zhu AX, Park JO, Ryoo BY, Yen CJ, Poon R, Pastorelli D, et al.; REACH Trial Investigators. Ramucirumab versus placebo as second-line treatment in patients with advanced hepatocellular carcinoma following first-line therapy with sorafenib (REACH): a randomised, double-blind, multicentre, phase 3 trial. Lancet Oncol. 2015 Jul;16(7):859-70.

2 Kudo M, Hatano E, Ohkawa S, Fujii H, Masumoto A, Furuse J, et al. Ramucirumab as second-line treatment in patients with advanced hepatocellular carcinoma: Japanese subgroup analysis of the REACH trial. J Gastroenterol. 2017 Apr;52(4):494-503.

3 Park JO, Ryoo BY, Yen CJ, Kudo M, Yang L, Abada PB, et al. Second-line ramucirumab therapy for advanced hepatocellular carcinoma (REACH): an East Asian and non-East Asian subgroup analysis. Oncotarget. 2016 Nov;7(46):75482-91.

4 Chau I, Park JO, Ryoo BY, Yen CJ, Poon R, Pastorelli D, et al. Alpha-fetoprotein kinetics in patients with hepatocellular carcinoma receiving ramucirumab or placebo: an analysis of the phase 3 REACH study. Br J Cancer. 2018 Jul;119(1):19-26.

5 Zhu AX, Kang YK, Yen CJ, Finn RS, Galle PR, Llovet JM, et al: REACH-2: a randomized, double-blind, placebocontrolled phase 3 study of ramucirumab versus placebo as second-line treatment in patients with advanced hepatocellular carcinoma (HCC) and elevated baseline alpha-fetoprotein (AFP) following first-line sorafenib. J Clin Oncol. 2018(suppl):abstract 4003. 